\title{
EL SISTEMA PARTICIPATIVO DE GARANTÍAS (SPG) COMO ESTRATEGIA ADMINISTRATIVA EN LOS TERRITORIOS RURALES CAFETEROS DEL CAUCA, COLOMBIA $^{*}$
}

\section{THE PARTICIPATORY GUARANTEE SYSTEM (SPG) AS AN ADMINISTRATIVE STRATEGY IN THE RURAL COFFEE-GROWING TERRITORIES OF CAUCA, COLOMBIA}

Recibido: 26 de septiembre de 2020

Evaluado: 2 de octubre de 2020

Aprobado: 18 de noviembre de 2020

\author{
Mayra Roxana Solarte Montoya** \\ Universidad de Brasilia \\ Orcid: https://orcid.org/0000-0001-7760-5890
}

\section{José Fernando Grass Ramírez ${ }^{\star \star \star}$}

Universidad Auntónoma de Chapingo

Orcid: https://orcid.org/0000-0001-6081-4581

Cómo citar este artículo: Solarte Montoya, M.R., y Grass Ramírez, J.F. (2021). EL SISTEMA PARTICIPATIVO DE GARANTÍAS (SPG) COMO ESTRATEGIA ADMINISTRATIVA EN LOS TERRITORIOS RURALES CAFETEROS DEL CAUCA, COLOMBIA. Revista Estrategia Organizacional, 10(1), https://doi.org/10.22490/25392786.4553

\footnotetext{
* $\quad$ Artículo de investigación.

** Magíster en Agronegocios, Universidad de Brasilia. Ingeniera Agroindustrial, Universidad del Cauca. Docente ocasional. Escuela de Ciencias Administrativas, Contables, Económicas y de Negocios - ECACEN. Universidad Nacional Abierta y a Distancia - UNAD. CEAD Popayán. Popayán, Colombia. Correo electrónico: mayrar.solarte@unad.edu.co.

*** Doctor en Problemas Económicos Agroindustriales, Universidad Autónoma Chapingo. Magíster en Desarrollo Rural, Universidad Javeriana. Especialista en Gerencia de Producción, Universidad Icesi. Ingeniero Químico, Universidad Nacional de Colombia. Profesor titular, Facultad de Ciencias Agrarias, Departamento de Agroindustria. Grupo de investigaciones para el Desarrollo Rural TULL - Universidad del Cauca. Popayán, Colombia. Correo electrónico: jfgrass@unicauca.edu.co.
} 


\section{RESUMEN}

Introducción: La economía campesina, la producción agroecológica, el relacionamiento entre organizaciones sociales de base, la resiliencia al cambio climático y la soberanía alimentaria, son prácticas y propuestas que se fortalecen en los territorios cafeteros del departamento del Cauca, Colombia. Sin embargo, en la primera escala territorial, la finca como sistema integrado de productivo, y en una segunda, el territorio; las dinámicas socio-económicas de la caficultura caucana son incipientes con relación a las certificaciones propias que permitan mitigar los problemas de comercialización de los productos agroecológicos y orgánicos, y fortalecer el relacionamiento directo entre productores y consumidores. Metodología: Es por ello, que en este estudio se plantea una ruta hacia un Sistema Participativo de Garantía (SPG), como estrategia y herramienta de certificación propia de los productos agroalimentarios locales donde se resalte la acción colectiva de las comunidades, se consoliden los lazos de confianza entre productores y consumidores, induciendo a nuevas teorías de gestión organizacional y territorial. Los caficultores del Cauca no solamente producen café, su economía local se enmarca en la comercialización de otros productos provenientes de sus fincas; y actualmente en los mercados convencionales donde generalmente participan, sus productos no están siendo reconocidos por los consumidores como elaborados con prácticas agroecológicas. Como referencia para generar la ruta, se sistematizaron dos experiencias en SPG, de los departamentos de Risaralda y Valle del Cauca, en Colombia, las cuales son lideradas por agricultores agroecológicos de la región. Resultados: En esta modalidad de Investigación-Acción-Participativa (IAP), se logró destacar factores de éxito para la implementación de un SPG y analizar cualitativamente el estudio de caso sistematizado. Para el caso de los caficultores caucanos, se genera la necesidad de explorar la identificación de circuitos comerciales que les permita vender sus productos en canales que reduzcan la participación de intermediarios y acerquen al productor con una venta basada en la confianza directa del consumidor.

Palabras clave: comercio justo, circuitos cortos, certificación, confianza, redes comerciales, administración estratégica. 


\begin{abstract}
Introduction: The peasant economy, agroecological production, the relationship between grassroots social organizations, resilience to climate change and food sovereignty, are practices and proposals that are strengthened in the coffee territories of the department of Cauca, Colombia. However, in the first territorial scale, the farm as an integrated production system, and in a second, the territory; The socio-economic dynamics of Cauca coffee farming are incipient in relation to the own certifications that allow mitigating the problems of marketing agroecological and organic products, and strengthening the direct relationship between producers and consumers. Methodology: That is why this study proposes a route towards a Participatory Guarantee System (SPG), as a strategy and certification tool for local agri-food products where the collective action of the communities is highlighted, ties are consolidated of trust between producers and consumers, inducing new theories of organizational and territorial management. Cauca coffee growers not only produce coffee, their local economy is part of the commercialization of other products from their farms; and currently in conventional markets where they generally participate, their products are not being recognized by consumers as made with agroecological practices. As a reference to generate the route, two experiences were systematized in SPG, from the departments of Risaralda and Valle del Cauca, in Colombia, which are led by agroecological farmers in the region. Results: In this Participatory Action-Research (PAR) modality, it was possible to highlight success factors for the implementation of a PGS and qualitatively analyze the systematized case study. In the case of Caucanos coffee growers, there is a need to explore the identification of commercial circuits that allow them to sell their products in channels that reduce the participation of intermediaries and bring the producer closer with a sale based on direct consumer confidence.
\end{abstract}

Keywords: fair trade, short circuits, certification, trust, commercial networks, strategic management. 


\section{INTRODUCCIÓN}

La presente investigación se contextualiza en la sistematización de dos (2) experiencias de Sistemas Participativos de Garantía que actualmente están en marcha en Colombia, como escenarios particulares, enfocándose en las apuestas de las organizaciones sociales, también como estrategia de valorización de los productos agroalimentarios y su articulación a las cadenas de valor desde los territorios. En ese sentido, estas experiencias son el foco para aplicar el método de estudio de caso, analizado a partir de la observación directa de las interacciones comunitarias interculturales.

La primera experiencia, en el departamento de Risaralda, donde los principales actores sin duda son las organizaciones campesinas e indígenas de esta región, el Instituto de Gestiones Ambientales - IIA, la Universidad Tecnológica de Pereira - UTP, y la Corporación Autónoma Regional de Risaralda - CARDER, estos cuatro actores se articulan en el SPG Risaralda y su principal espacio de comercialización es el Mercado Agroecológico de la UTP Alimentos para la vida; la segunda experiencia, en el departamento del Valle del Cauca, la Red de Mercados Agroecológicos Campesinos del Valle del Cauca "Alimentos de vida", es una organización que articula los distintos mercados agroecológicos campesinos del Valle del Cauca, se encuentra integrada por 58 organizaciones de productores, con aproximadamente 300 familias vinculadas en forma directa participando en veinte $\mathbf{2 0}$ mercados activos en el Valle del Cauca y norte del Cauca. Estas dos experiencias son referencias a nivel nacional de empoderamiento de las organizaciones sociales para comercializar sus productos sin intermediarios y ofrecer a los consumidores productos sanos y de calidad, donde se teje la economía social y solidaria en tiempos de incertidumbre.

Cabe resaltar que, aunque existen entidades certificadoras, en la mayoría de casos, se dificulta que los pequeños productores, como es el caso de los caficultores del Cauca, puedan contar con estos sellos de tercera parte, los cuales no necesariamente son garantía de un comercio justo, lo que sí implica un alto costo que restringe las oportunidades; y en el caso de productores orgánicos y agroecológicos, un largo periodo de transición desde la producción convencional.

Como respuesta al modelo de certificación por terceros, se han generado movimientos alternativos que buscan certificar procesos y productos sin tener que recurrir a otros organismos de 
control, a partir de dinámicas de construcción social basados en la organización y en la confianza entre agricultores y consumidores. Este modelo de certificación se ha llevado a cabo en diversos países como Brasil y Perú, entre otros, trayendo resultados a resaltar para las pequeñas unidades familiares que desarrollan actividades agrícolas de forma ecológica, orgánica o agroecológica; este proceso de certificación se conoce como Sistema Participativo de Garantía (SPG).

En Colombia, los SPG se han desarrollado en los departamentos del Valle del Cauca, Risaralda, Antioquia y la Costa Atlántica construyendo una cultura de producción ecológica basada en la confianza, generando además valor agregado (CARDER-UTP, 2012), aunque en la actualidad en el país no existe un marco regulatorio que avale los SPG, este trabajo es impulsado por organizaciones no gubernamentales, movimientos agroecológicos, organizaciones de productores, familias de agricultores ecológicos a pequeña escala con sistemas de producción diversificados con iniciativas de abastecer a los mercados locales (IFOAM, 2013).

\section{CONTENIDO}

Los elementos conceptuales que soportan esta investigación se evidencian básicamente los conceptos de Sistemas Participativos de Garantía (SPG), Sistemas Agroalimentarios Localizados (SIAL), Acción Colectiva y Sustentabilidad.

Sistemas Participativos de Garantía (SPG). El desarrollo de los mercados nacionales y locales de productos orgánicos, agroecológicos, sanos, va un poco más allá del establecimiento físico de los puntos de venta, además de regirse por la oferta y la demanda de los productos. En algunos casos, los productores que emplean prácticas orgánicas en su producción, aducen un alto costo en el valor de la certificación de tercera parte. El costo de la certificación de este tipo, ha llevado a algunos movimientos sociales a desarrollar alternativas de garantía para los consumidores de los mercados nacionales y locales, en aras de ser mayormente accesibles a quienes los producen y así minimizar el efecto sobre los precios (IICA, 2010).

Como sistema de verificación alternativo y complementario a la certificación independiente de tercera parte, los SPG no sólo garantizan la credibilidad del producto ecológico sino que también están relacionados con enfoque de comercialización local y alternativa, fortaleciendo la participación directa de los agricultores, consumidores y otros actores en el proceso de 
verificación, estableciendo y favoreciendo relaciones comerciales directas o de corta distancia, es decir, fortaleciendo los Circuitos Cortos de Comercialización.

Los productos que provienen de los SPG, son vendidos a consumidores que demandan principalmente productos particulares, locales y que cuyas características de producción aún no se corresponde con procedimientos de certificación para la exportación. Es así como han surgido iniciativas para la generación de garantías de la calidad de estos productos con base en formas alternativas de certificación como el SPG (Comunidad Andina, 2011).

La certificación participativa es una propuesta alternativa, donde se construye bajo las premisas del enfoque socio-territorial, que integra productores y consumidores. Según Torremocha (2012: 94):

Así, aplicados en cada territorio, los Sistemas Participativos de Garantía se convierten en herramientas idóneas para construir, desde el ámbito agrario, un modelo de sistema basado en el intercambio social, cultural y económico que recoge todos los preceptos de la agroecología, como ciencia, pero también como disciplina aplicada. Por ende, se puede considerar que constituyen una herramienta con gran potencial para reproducir y multiplicar prácticas concretas de corte agroecológico, y ello en múltiples ámbitos del agroecosistema entendido en la concepción amplia que le otorga la agroecología aplicada como pueden ser la producción, el consumo o la comercialización. Esto los convierte en un instrumento idóneo para la aplicación de una agroecología política.

Un Sistema Participativo de Garantía se caracteriza por el control social y por la responsabilidad solidaria, que generan credibilidad a las diferentes realidades sociales, culturales, políticas, institucionales, organizacionales y económicas, las cuales son reforzadas por algunas características propias, como la confianza, la descentralización, la participación, la organización de base, la transparencia, la formación en red, la adecuación de la agricultura familiar y el proceso pedagógico (Centro Ecológico de Brasil, 2014). La certificación participativa puede contribuir a la valorización de los productos ecológicos, pues consiste en un momento importante de reflexión sobre el trabajo de los grupos, que debe ser aprovechando para una mayor grado de sustentabilidad de la agroecología en las fincas y como apuesta al desarrollo de la agroecología. Por tanto, aquí 
está más un desafío para los grupos, consejos de ética, núcleos y toda la red, en la lucha por un mundo más justo, solidario y saludable (Rede Ecovida Brasil, 2004).

Sistemas Agroalimentarios Localizados - SIAL. EI SIAL es un modelo de organización productiva, en búsqueda del análisis de la relación territorio-actores-técnicas-productos agroalimentarios tradicionales (Grass Ramírez et al., 2018). Los SIAL se definen como (...) organizaciones de producción y de servicios, asociadas por sus características y su funcionamiento a un territorio específico: el medio, los productos, las personas, sus instituciones, su saber-hacer, sus comportamientos alimentarios y sus redes de relaciones, se combinan en un territorio para producir una forma de organización agroalimentaria en una escala espacial dada (Muchnik y Sautier, 1998, p.4), apuntando a la comprensión de las dinámicas y la diversidad de los procesos de anclaje territorial y su evolución en el tiempo (Fournier y Muchnik, 2012).

Acción colectiva. La lógica de la acción colectiva planteada por Olson (1965), considera algunas restricciones, "es más probable que los grupos más reducidos emprendan una acción colectiva, en comparación con los grupos más numerosos, (...) o a menos que exista coerción o algún otro dispositivo para hacer que los individuos actúen a favor de su interés común". Los grupos pequeños poseen una fuente adicional de incentivos selectivos, aprecian el compañerismo, el respeto por los demás. Si algún factor como la heterogeneidad social, reduce el consenso, la acción colectiva se vuelve menos probable, y si pese a todo existe una acción colectiva, tiene el costo adicional de conciliar y arbitrar las diferentes opiniones, sobre todo para los dirigentes de la organización o de la asociación de intereses en cuestión. Por otra parte, Ostrom (2000) plantea la lógica de la acción colectiva como concepto relacionado en los modelos que definen a los individuos cuando intentan lograr beneficios colectivos, la autora define en estos modelos, el problema del gorrón (free rider), es decir, cuando una persona no puede ser excluida de los beneficios que otros buscan, está motivada a no contribuir en el esfuerzo común y a 'gorronear' los esfuerzos de los otros, por consiguiente, individuos racionales produciendo resultados irracionales y en algunas circunstancias beneficiándose con el trabajo ajeno.

Con el enfoque SIAL se pueden definir al menos dos etapas de acción colectiva (Boucher, 2004). La primera, relacionada con la acción colectiva estructural, la cual se refiere a los encuentros e intercambios favorables de los procesos organizativos y aprendizajes colectivos, mediante una 
asociación, cooperativa u otro modelo de agremiación. Se pueden realizar economías de escala, generar interacción política y económica con los clientes, proveedores, presencia y gestión de las instituciones, brinda a los participantes visibilidad y representatividad, estimula el liderazgo y el capital social.

Los límites de esta acción colectiva estructural, están en la regulación y el control de la oferta en términos de calidad o cuando se profundizan asimetrías de poder entre los miembros. Para Grass Ramírez et al. (2018), las características del territorio, la identidad de las comunidades que allí habitan y las capacidades de las instituciones presentes producen particularidades sobre la acción colectiva estructural.

Para Boucher (2004), la segunda etapa de acción colectiva, la funcional, se remite a la construcción de recursos territorializados relacionados con la calidad de un bien o servicio, mediante la obtención de sellos de calidad, marcas colectivas, denominación de origen u otro tipo de indicación geográfica, permitiendo consolidar la diferenciación de los productos que se desarrollan en el territorio.

Sustentabilidad. Este concepto propone ajustes a la agricultura convencional para hacerla ambientalmente, socialmente y económicamente más viable y compatible, orientado hacia la reducción o eliminación de agroquímicos a través de cambios en el manejo, que aseguran la adecuada nutrición y protección de las plantas a través de fuentes de nutrientes orgánicos y un manejo integrado de plagas, respectivamente (Altieri, 2001). Según Toledo (1996), este concepto recorre todos los ámbitos políticos, financieros, sociales, diplomáticos, culturales, científicos del mundo contemporáneo como un espectro multifacético, integrando las dimensiones ecológicas sociales y económicas del desarrollo y toma en cuenta a las generaciones futuras, además contiene seis pilares del desarrollo comunitario sustentable: la toma de control de su territorio, el uso adecuado o no destructivo de los recursos naturales, el control cultural, la toma de control social, la regulación de los intercambios económicos, y por último, la toma de control político.

De otro lado, para Cicaficultura (2015), los modelos de producción sustentable en los territorios cafeteros caucanos, están orientados hacia: la producción de acuerdo a las condiciones agroecológicas de cada territorio, la producción diversificada con principios agroecológicos, 
doi: https://doi.org/10.22490/25392786.3644

autonomía en la producción, producir alimentos sanos y nutritivos, realizar procesos de adaptación al cambio climático, menor dependencia externa y prácticas tradicionales o ancestrales.

\section{METODOLOGÍA}

La metodología estuvo apoyada en la sistematización de experiencias, para ello se hizo importante la elaboración de un instrumento metodológico como guía para las entrevistas semi-estructuradas que se realizaron a treinta (30) actores relacionados con las experiencias abordadas en el año 2019, entre productores, consumidores, líderes de las asociaciones de producción agroecológica, colaboradores de logística, gestores de las instituciones públicas y privadas, y estudiantes universitarios. El instrumento incorporó ejes de análisis que buscaron comprender y abordar los objetivos específicos relacionados con el conocer el origen del proceso, evaluar las condiciones de ingreso de los productores, considerar las normas que rigen los procesos, identificar las dificultades en la consolidación de la experiencia, identificar el apoyo institucional y analizar la participación del consumidor, entre otras. La aplicación del instrumento a los actores de las dos (2) experiencias, permitió identificar los elementos comunes y diferentes entre éstas. Las experiencias permitieron reconocer desde la práctica, cómo se crea, desarrolla y consolida un SPG, esta información resultó de gran importancia para atemperar la información teórica, y establecer las etapas posteriores para proponer el inicio de un SPG para los caficultores caucanos. En la sistematización, el objeto de conocimiento es la experiencia de intervención, o sea la práctica, y tiene un sentido siempre de carácter transformador, "...no sistematizamos para informarnos de lo que sucede y seguir haciendo lo mismo, sino para mejorar, enriquecer, transformar nuestras prácticas" (Jara, 2001).

El estudio de caso sistematizado, contribuye a la adquisición del conocimiento de los fenómenos individuales, grupales, organizacionales, sociales y políticas; los estudios de caso también se encuentran en los análisis económicos, en la estructura de un determinado sector industrial, en la economía de una ciudad o en una región a ser investigada. Además, en cualquier tipo de situación, la necesidad diferenciada de los estudios de caso, surgen del deseo de entender los fenómenos sociales complejos (Yin, 2010, p.24). Cabe mencionar, que la investigación cualitativa es el principal medio para el alcance de los objetivos que tuvo esta investigación, pues como afırma Godoy (2006), el estudio de caso debe ser centrado en una situación o evento en particular, cuya importancia viene de lo que éste pueda revelar sobre el fenómeno objeto de la investigación. 


\section{RESULTADOS}

Si bien esta investigación refleja los elementos para construir una ruta hacia un Sistema Participativo de Garantía (SPG) para los caficultores caucanos, esta propuesta se gesta desde las dinámicas territoriales donde se evidencia el enfoque socio-territorial, donde uno de los escenarios, son sin duda, los mercados interculturales y solidarios. Para Cicaficultura (2015), los mercados multiculturales, los cuales se instauran como una iniciativa de control cultural, donde se llevan a cabo y se dinamizan el intercambio de productos, las sinergias entre etnias, las diversas formas de hacer y pensar y donde de igual manera, se evidencian las inconformidades, en tanto es posible identificar y expresar las injusticias en relación al costo de producción y el valor de venta. Además, para los caficultores caucanos, el día de mercado se concibe como el escenario de actividades culturales, (bailes, fiestas, juegos, entre otras) intercambio de productos entre los diferentes pisos climáticos, intercambio de conocimientos, puesta en marcha y fortalecimiento de procesos organizativos, políticos y culturales.

En los procesos de creación de los SPG, la acción colectiva de los productores ha resultado determinante, sin embargo el apoyo institucional, especialmente de instituciones públicas, ha permitido su consolidación, este apoyo ha consistido especialmente en conseguir espacios para la comercialización de los productos, tales como espacios en universidades públicas, parques, plazas y patios, es decir, espacios públicos abiertos. También, estas instituciones han sido claves en la divulgación y promoción de estos mercados a través de sus plataformas virtuales y redes sociales.

La consolidación de los SPG ha sido gradual, en ese proceso se conservan como integrantes una fracción pequeña de los productores que emprenden las actividades. La vinculación de nuevos participantes generalmente es muy limitado y en algunos casos cerrado. Las normas generalmente se construyen y ponen en marcha a través del tiempo, la existencia de un grupo de control y vigilancia, es la forma de autorregulación que garantiza el cumplimiento de la calidad de los productos. La existencia de visitas de los clientes y/o consumidores a las fincas productoras, les permiten a ellos mismos confirmar las características de interés en los productos. Generalmente los precios de los productos se fijan para todo el año, este aspecto se vuelve en un factor diferenciador adicional para los consumidores, además de las características agroecológicas de los productos a ofertar. Aunque la mayor parte de los productores de las dos (2) experiencias sistematizadas se encuentran en fases de transición en sus sistemas productivos, se reconocen gradientes importantes en la evolución agroecológica y en los niveles de organización de los productores, 
en cuanto a la producción, algunos cuentan con niveles avanzados en producción agroecológica, pero la mayor parte de ellos continúan incorporando algunos insumos propios de la producción convencional, en ese sentido, se identifica la ausencia de mayores apoyos y acompañamientos técnicos, para lograr evolucionar en este tipo de iniciativas productivas y comerciales.

Con las anteriores premisas y los resultados obtenidos a partir del instrumento que se diseñó y validó para sistematizar las dos (2) experiencias, en el cual se abordaron los elementos: diseño de producción, estructura del sistema y organización, certificación, normas y reglamentación, valor agregado, gastos administrativos y comercialización; se puede visibilizar las similitudes y diferencias de las experiencias abordadas como estudio de caso.

Diseño en la producción. En las características en el diseño en la producción de las experiencias estudiadas, se abordaron preguntas referentes a quiénes son los miembros del sistema, cómo ingresan al sistema y cuáles son las reglas de juego en la producción, para que una finca o productor pueda participar del SPG.

Tabla 1. Elementos del diseño de producción.

\begin{tabular}{|c|c|}
\hline Elementos & Características en el diseño de producción \\
\hline Similitudes & $\begin{array}{l}\text { Los actores del sistema son personas físicas, generalmente productores y consumidores, así como } \\
\text { entidades públicas o privadas que participan como facilitadores del proceso. } \\
\text { Reglas del juego: } \\
\text { 1. Los productores deben hacer parte de una asociación adscrita a la Red de Mercados. } \\
\text { 2. Se debe realizar una solicitud formal al comité de diálogo del SPG. } \\
\text { 3. Si no cumple con el primer requisito, debe hacer una solicitud a la junta directiva de la Red de } \\
\text { Mercados, cumpliendo con los principios agroecológicos y en algunos casos, ofertando productos } \\
\text { diferentes a los que comercialicen actualmente en los mercados. } \\
\text { 4. Diseñar los instrumentos de verificación de conformidad para aplicar en los predios (radiografía del } \\
\text { sistema productivo) en términos de sustentabilidad } \\
\text { 5. El Comité de Diálogo visita a la finca del solicitante y aplica los instrumentos de productor a productor. } \\
\text { 6. Se hacen las recomendaciones respectivas para una segunda visita. } \\
\text { 7. En caso de ser aprobado, se firma el acuerdo de vida del SPG } \\
\text { 8. Las fincas que participan del SPG pueden estar en transición agroecológica. } \\
\text { 9. No todos los proveedores del mercado (productores) hacen parte del SPG, pero sí se basan en los } \\
\text { principios agroecológicos. }\end{array}$ \\
\hline Diferencias & No se presentó ninguna diferencia en la temática de producción. \\
\hline
\end{tabular}

Fuente: elaboración propia 
Estructura del sistema y organización. La estructura del sistema y la organización de los productores estuvo orientada en conocer cómo está organizada la estructura del SPG, cuál es el tiempo estimado para crear un SPG, en conocer si existe algún protocolo para ingresar al SPG y cuáles son los lineamientos, quiénes lo pueden conformar, si existe algún manual de funciones para quienes participan en el SPG, cuáles son las normas internas de regulación entre los miembros del SPG, qué instituciones han apoyado, cuál es el vínculo entre proveedores y colaboradores, y si existe capacitación técnica por parte de alguna entidad privada o pública.

Tabla 2. Elementos de la estructura del sistema y organización.

\begin{tabular}{|l|l|}
\hline \multicolumn{1}{|c|}{ Elementos } & \multicolumn{1}{|c|}{ Características del sistema y organización } \\
\hline Similitudes & $\begin{array}{l}\text { La estructura del SPG está organizada por un Comité de Diálogo (por lo menos un } \\
\text { representante de cada grupo de actores), productores, consumidores, representantes de } \\
\text { entidades públicas y/o privadas. } \\
\text { Protocolo para ingresar al SPG: }\end{array}$ \\
\hline 1. Creación de los comités de diálogo de la organización y del mercado. \\
2. Capacitación de los comités de diálogo y entrega de los documentos. \\
3. Solicitud de las personas interesadas en el SPG. \\
4. Estudio de la solicitud y los documentos de la persona interesada. \\
5. Visita a la familia/finca por parte del Comité de Diálogo. \\
6. Elaboración del informe y concepto por el Comité de Diálogo de la Organización. \\
7. Recepción de la información por parte del Comité de Diálogo de Mercado Agroecológico \\
Campesino y traslado a la Red. \\
8. Estudio por parte de la red y otorgamiento del certificado y sello. \\
9. Ejecución y seguimiento.
\end{tabular}

Fuente: elaboración propia 
Certificación, normas y reglamentación. Esta sesión estuvo orientada en preguntas que abordaron temáticas como la reglamentación de la certificación de confianza, en cómo se entiende, quién las regula, cuáles son los principales pilares de la certificación desde los procesos locales y si existe alguna entidad que avale los SPG. Estas preguntas fueron realizadas con el fin de conocer el proceso que las experiencias han llevado a cabo, como alternativa de certificación.

Tabla 3. Elementos de la certificación, normas y reglamentación.

\begin{tabular}{|c|c|}
\hline Elementos & Características de la certificación, normas y reglamentación \\
\hline Similitudes & $\begin{array}{l}\text { - La certificación de confianza, es valorada a través de los mercados y las tiendas } \\
\text { agroecológicas, presentándose ante los consumidores una propuesta diferente a } \\
\text { las certificaciones de tercera parte. } \\
\text { - Los sellos propios se visibilizan en los empaques de los productos generando } \\
\text { confianza al consumidor, y también son útiles para realizar extensión } \\
\text { agroecológica, es decir, que los productores agroecológicos intercambien saberes } \\
\text { con productores convencionales. } \\
\text { - La certificación de tercera parte es más costosa que la certificación de confianza. } \\
\text { - La certificación de confianza es una cuestión de ética, mientras que en una } \\
\text { certificación de tercera parte lo visitan cada año y con previo aviso. } \\
\text { - Los principios agroecológicos son el de producir sanamente, se produce con ética } \\
\text { porque alguien se lo va a comer, y no porque tiene que tener ciertas condiciones } \\
\text { para poder ser comercializado con un sello de tercera parte. } \\
\text { - Los mercados campesinos deben ser apoyados por una política pública en cada } \\
\text { municipio. } \\
\text { - La certificación de confianza es avalada por la IFOAM, pero quienes dan la } \\
\text { certificación a las fincas, es la misma red de productores agroecológicos a través } \\
\text { del Comité de Diálogo. } \\
\text { - Falta resignificar el sentido del SPG en las dinámicas de comercialización, que se } \\
\text { valoren las prácticas que se han tejido alrededor de la certificación, tanto por parte } \\
\text { de los productores como de los consumidores. El objetivo no es el sello, la esencia } \\
\text { es la confianza en el otro. } \\
\text { - La certificación es para la finca como unidad productiva, no por cada producto } \\
\text { como en la certificación de tercera parte. }\end{array}$ \\
\hline Diferencias & $\begin{array}{l}\text { SPG Risaralda: Existen dos sellos, uno es para la certificación de confianza } \\
\text { (cumplimiento total para SPG) y el otro en transición (cumplimiento parcial para SPG). } \\
\text { SPG Valle del Cauca: Existe un sello único de garantía de productos agroecológicos. }\end{array}$ \\
\hline
\end{tabular}

Fuente: elaboración propia 
Valor agregado. En esta temática se visibilizan cuáles son las estrategias de valor agregado generadas por los participantes del SPG y cuáles son las instituciones que ayudan a apalancar procesos de transformación agroindustrial rural, además de mencionar las materias primas a las cuales se les agrega mayor valor.

Tabla 4. Elementos del valor agregado.

\begin{tabular}{|l|l|}
\hline \multicolumn{1}{|c|}{ Elementos } & \multicolumn{1}{c|}{ Características del valor agregado } \\
\hline Similitudes & $\begin{array}{l}\text { Algunas de las instituciones que apalancan procesos de transformación agroindustrial son el } \\
\text { Servicio Nacional de Aprendizaje - SENA, e Instituciones Educativas Rurales. } \\
\text { - Las materias primas a las cuales se les genera mayor valor agregado son el café, guayaba, leche, } \\
\text { carne y verduras. }\end{array}$ \\
\hline Diferencias & $\begin{array}{l}\text { Las estrategias de valor agregado en la mayoría de casos son realizadas en las fincas de los } \\
\text { productores. En algunos casos, hay plantas de tratamiento en la zona rural que maquilan o acopian } \\
\text { materia prima para transformación, elaboración de productos como mermeladas, bocadillos, } \\
\text { cárnicos y lácteos. }\end{array}$ \\
\hline
\end{tabular}

Fuente: elaboración propia

Gastos administrativos. Los gastos administrativos hacen referencia a cuáles son los ingresos y/o utilidades para el productor cuando se disminuyen los intermediarios, cómo se asumen estos gastos, y la manera de sostenimiento del SPG.

Tabla 5. Elementos de los gastos administrativos.

\begin{tabular}{|c|c|}
\hline Elementos & Características de los gastos administrativos \\
\hline Similitudes & $\begin{array}{l}\text { - Aunque no se tiene cuantificado exactamente cuántos ingresos le quedan al productor, sí se } \\
\text { establece una diferencia al vender los productos en un mercado convencional donde no valoran el } \\
\text { valor agregado que tienen los productos, mientras que en los mercados agroecológicos se eliminan } \\
\text { los intermediarios y va a quedar un margen de ganancia más alto. } \\
\text { - Los mercados agroecológicos funcionan como corporación sin ánimo de lucro. } \\
\text { - Cada mercado lleva un registro de los productos que más se comercializan. Esa información es } \\
\text { confidencial. }\end{array}$ \\
\hline Diferencias & $\begin{array}{l}\text { - En algunos mercados campesinos agroecológicos, los gastos administrativos se pagan del fondo rotario, } \\
\text { que se establece a partir de cuotas a los productores o de actividades que hacen entre asociados. } \\
\text { - El sostenimiento del SPG generalmente es de autogestión, en algunos casos reciben apoyos } \\
\text { institucionales para algunos gastos como transporte, tanto para los productores que participan } \\
\text { en los mercados como para los participantes del Comité de Diálogo quienes realizan las visitas de } \\
\text { verificación. } \\
\text { - Algunas fincas, reciben visitas para conocer las prácticas agroecológicas que se hacen en cada una } \\
\text { de ellas, recibiendo a cambio, apoyos monetarios por los servicios que se brindan. }\end{array}$ \\
\hline
\end{tabular}

Fuente: elaboración propia 
Comercialización. En esta temática se abordaron preguntas orientadas a mencionar las ventajas y desventajas del proceso de comercialización alternativa, las etapas para la consolidación de estas estrategias, los canales de comercialización, y finalmente, mencionar si los SPG son una fuente de generación de ingresos sostenibles para las fincas agroecológicas.

Tabla 6. Elementos de la comercialización.

\begin{tabular}{|c|c|}
\hline Elementos & Características de la comercialización \\
\hline Similitudes & $\begin{array}{l}\text { - Los precios de los productos va a depender de dónde se comercialicen (tipo de mercado, ciudad, } \\
\text { entre otros aspectos) } \\
\text { - Los productos agroecológicos no necesariamente son más costosos que los productos } \\
\text { convencionales. } \\
\text { - La confianza con el consumidor se fortalece invitándole a las fincas. } \\
\text { - Al finalizar los mercados, entre productores hay intercambio de productos (trueque). } \\
\text { - La prioridad de los productores agroecológicos es el autoconsumo, y posteriormente, la } \\
\text { comercialización de los productos que no se consumen en la finca. } \\
\text { - La regularidad de los mercados agroecológicos es beneficiario para los ingresos de las familias } \\
\text { que participan. } \\
\text { - Los canales de comercialización para la mayoría de productores que realizan prácticas } \\
\text { agroecológicas, son los mercados campesinos, pero en algunos casos, también comercializan } \\
\text { en tiendas agroecológicas de las ciudades. }\end{array}$ \\
\hline Diferencias & No se presentó ninguna diferencia en la temática de comercialización. \\
\hline
\end{tabular}

Fuente: elaboración propia

\section{DISCUSIÓN}

Con base en los elementos arrojados en las anteriores tablas, se construye la propuesta para la ruta hacia un Sistema Participativo de Garantía - SPG, la cual se desarrolló bajo el método de Investigación-Acción-Participativa (IAP), en donde se visibilizan diez (10) pasos, los cuales podrán ejecutarse de manera secuencial o simultánea, buscando responder de manera lógica al objetivo final que es la creación de un SPG para los caficultores del departamento del Cauca. Estos pasos fueron elaborados a partir de las experiencias de los SPG Risaralda y Valle del Cauca.

Paso 1: Conocer las experiencias en SPG, sistematizarlas y socializarlas. Conocimiento en campo, sistematización de experiencias de SPG a nivel nacional y socialización a la comunidad interesada. Esta etapa requiere de un trabajo de campo, donde los investigadores y comunidad participen en el intercambio de experiencias y socialización de los conocimientos adquiridos en otras zonas del país. 
Paso 2: Revisión bibliográfica. Desarrollo teórico alrededor del Sistema Participativo de Garantía, el cual requiere una revisión de información secundaria referenciando bibliografía referente a los SPG, donde los investigadores acompañen actividades junto a las comunidades, con el ánimo de incorporar los aportes de los actores sociales comunitarios a los referentes teóricos.

Paso 3: Construcción de los lineamientos y plan de vida del SPG. Generación de protocolos necesarios para un proceso de certificación propia basados en la confianza. Para ello, es fundamental realizar jornadas de trabajo comunitario con los productores que estén en transición agroecológica, donde se construyan los lineamientos del SPG, y se elabore el plan de vida.

Paso 4: Conformación de grupos de trabajo, elaboración y aplicación de instrumentos metodológicos generados desde la comunidad. Conformación participativa de los núcleos locales y elaboración de instrumentos de verificación. Se conforman los grupos entre los participantes (Comité de Diálogo, junta directiva, evaluadores, entre otros) y se elaboran los instrumentos para caracterización de fincas, acta de compromiso, respaldo de confianza y verificación de garantía, aunando esfuerzos entre academia-comunidad, donde posteriormente son aplicados en las fincas con el fin de generar criterios para otorgar la certificación participativa.

Paso 5: Aplicación y validación de los instrumentos metodológicos para caracterización de fincas. Se aplica y valida el instrumento para caracterización de las fincas con base en un formato donde se diligencia la información básica de la finca, se hace cartografía del predio, se abarcan preguntas relacionadas a la producción agrícola, producción animal, transformación y artesanías.

Paso 6: Aplicación y validación del instrumento para concepto de certificación. Los integrantes del Comité de Diálogo son quienes visitan las fincas interesadas en certificarse y realizan el concepto de certificación de las fincas.

Paso 7: Jornadas de IAP para fortalecer el proceso organizativo comunitario y diseñar el sello de garantía. Se realiza la retroalimentación, evaluación de los pasos anteriores y la consolidación de iniciativas comunitarias alrededor de la certificación de confianza, además de tejer comunitariamente el diseño del sello de garantía. 
Paso 8: Certificación de confianza a las fincas que cumplan con las especificaciones del SPG. Apropiación del sello distintivo y certificación de confianza a las fincas, el cual será entregado a las experiencias que hayan sido valoradas con conformidad por el Comité de Diálogo del SPG.

Paso 9: Fortalecimiento de mercados agroecológicos existentes y promoción de la gira de consumidores a las fincas certificadas. Fortalecimiento de iniciativas locales de comercialización en red, que busquen un comercio justo y su propia certificación. Además, promocionar la gira de consumidores, fortaleciendo los lazos de confianza a través de la visita de los consumidores a las fincas y proveedores de los mercados agroecológicos.

Paso 10: Validar la ruta metodológica para generar el SPG. Socialización de la metodología a los participantes del proceso y aprobación de la ruta, además de la generación de propuestas para el sostenimiento de la certificación de confianza.

\section{CONCLUSIONES}

Para la consolidación de un Sistema Participativo de Garantía, indudablemente se necesita el empoderamiento comunitario para fortalecer las economías locales, no sin ello, desconocer que las instituciones públicas y/o privadas, y la academia, puedan apoyar estos procesos, trabajando en conjunto para que los SPG pasen de ser solamente un sistema de certificación alternativo, a espacios donde se manifiesten posiciones y formas de vida de los territorios rurales.

Los productos en transición agroecológica provenientes de las fincas cafeteras del departamento del Cauca, necesitan un canal de comercialización alternativo donde el productor sea quien venda directamente al consumidor, por ello, es importante que las organizaciones de base se empoderen de los procesos que se entretejen en los mercados agroecológicos y haya más protagonismo en otros espacios como tiendas comunitarias, espacios en ferias agropecuarias y de universidades, mercados itinerantes y oferta de sus productos agropecuarios a través de plataformas virtuales. Además de ello, crezca el interés por participar en procesos alternativos de certificación propia, donde la confianza y solidaridad sean las bases sólidas éticas y políticas de estos procesos. 
Con la propuesta de los diez (10) pasos para la construcción de la ruta hacia un SPG como estrategia territorial de comercialización para los caficultores caucanos, la disminución de la brecha entre el productor y consumidor, y la generación de confianza entre estos dos actores locales, sin duda, es una apuesta de los procesos de acción colectiva que determinan el fortalecimiento de los Sistemas Agroalimentarios Localizados (SIAL), mediante estrategias de diferenciación y valor agregado de los productos del territorio, apuntando además a la soberanía alimentaria, la dinamización de la comercialización local y a las certificaciones alternativas provenientes de la postura política y el empoderamiento de las organizaciones sociales rurales y urbanas

\section{REFERENCIAS}

Altieri, M. A. (2001). Agroecologia: principios y estrategias para disenar sistemas agrarios. En Agroecología: principios y estrategias para diseñar una agricultura que conserva recursos naturales y asegura la soberanía alimentaria.

Boucher, F. (2004). Enjeux et difficulté dúne strategie collective d'activation des concentrations d'Agro-Industries Rurales, le cas des fromogeries rurales de Cajamarca, Perú. Université de Versailles San Quentin en Yvelines. Versailles:UFR Sciences Sociales et Humanities.

Centro Ecológico de Brasil. (2014). Cartilha: Sistema Participativo de Garantia. Simplificando seu entendimento.

Comunidad Andina. (2011). Agricultura familiar agroecológica campesina en la comunidad andina. Una opción para mejorar la seguridad alimentaria y conservar la biodiversidad.

Corporación Autónoma Regional de Risaralda - CARDER. Universidad Tecnológica de Pereira - UTP. (2012). Sistema Participativo de Garantías Risaralda. UTP, Pereira.

Federación Internacional de Movimientos de Agricultura Orgánica - IFOAM (2013). Sistemas Participativos de Garantía. Estudios de caso en América Latina.

Fournier, S., y Muchnik, J. (2012). El enfoque SIAL (Sistemas Agroalimentarios Localizados) y la activación de recursos territoriales. Revista Agroalimentaria, 18(34), 133-144. 
Godoy, A. (2006). Estudo de caso. In: Silva, A. B. et al. Pesquisa qualitativa em estudos organizacionais, paradigmas, estratégias e métodos. São Paulo, Brasil.

Grass Ramírez, J. F., Cervantes Escoto, F., y Palacios Rangel, M. I. (2018). Los sistemas agroalimentarios localizados: Rescate del patrimonio quesero en México. Popayán: Universidad del Cauca.

Instituto Interamericano de Cooperación para la Agricultura - IICA (2010). Sistemas de garantía para productos orgánicos en mercados locales y nacionales.

Jara, O. (2001). Dilemas y Desafíos De La Sistematización De Experiencias. Costa Rica.

Olson, M. (1965). The Logic Collective Action: Publics Good and the Theory of Groups. Cambridge: Harvard University Press.

Ostrom, E. (2000). El Gobierno de los Bienes Comunes: La Evolución de las Instituciones de Acción Colectiva. México: Fondo de Cultura Económica.

Proyecto Centro de Investigación, Promoción e Innovación Social para el Desarrollo de la Caficultura Caucana - CICAFICULTURA. (2015). Estrategias para la inclusión del enfoque socio-territorial de la caficultura caucana. Popayán, Cauca, Colombia.

Rede de Agroecología Ecovida Brasil (2004). Caderno de formação: certificação participativa de produtos ecológicos. Florianópolis: Rede Ecovida de Agroecologia, 2004, 48p.

Toledo, V. M. (1996). Red de Ecología Social. Principios etnoecológicos para el desarrollo sustentable de comunidades campesinas e indígenas. México: CLAES.

Torremocha, E. (2012). Los sistemas participativos de garantía. Herramientas de definición de estrategias agroecológicas. Agroecología, 6, 89-96.

Yin, R. (2010). Estudo de caso: planejamento e métodos. Tradução Ana Thorell; revisão técnica Cláudio Damacena. 4. ed. Porto Alegre: Bookman. p.24, 124, 133. 